Pacific Journal of Mathematics

COMMUTATIVE CANCELLATIVE SEMIGROUPS WITHOUT 


\title{
COMMUTATIVE CANCELLATIVE SEMIGROUPS WITHOUT IDEMPOTENTS
}

\author{
H. B. Hamilton, T. E. Nordahl and T. Tamura
}

A commutative cancellative idempotent-free semigroup (CCIF-) $S$ can be described in terms of a commutative cancellative semigroup $C$ with identity, an ideal of $C$, and a function of $C \times C$ into integers. If $C$ is an abelian group, $S$ has an archimedean component as an ideal; $S$ is called an $\bar{\Re}$-semigroup. A CCIF-semigroup of finite rank has nontrivial homomorphism into nonnegative real numbers.

1. Introduction. In this paper, a commutative cancellative semigroup without idempotent is called a CCIF-semigroup (in which, by "IF" we mean "idempotent-free") and a commutative cancellative semigroup with identity is called a CCI-semigroup. In particular, an $\mathfrak{N}$-semigroup is an archimedean CCIF-semigroup. The structure of $\Re$-semigroups has been much studied $[1,2,3,6,7,8]$ and also it is well known that every CCIF-semigroup is a semilattic of $\mathfrak{R}$-semigroups. In this paper CCIF-semigroups will be studied by means of the representation by the generalized $\mathscr{F}_{-}$and $\varphi$-functions and also through homomorphisms into the nonnegative real numbers.

Throughout this paper, $\boldsymbol{R}$ denotes the set of real numbers; $R$ the set of rational numbers; $\boldsymbol{R}_{+}$the set of positive real numbers; $\boldsymbol{R}_{+}^{0}$ the set of nonnegative real numbers; $Z_{+}$the set of positive integers and $Z_{+}^{0}$ the set of nonnegative integers. Each of these is a semigroup under the usual addition. If $S$ is a semigroup and if $X$ is a subsemigroup of the group $\boldsymbol{R}$, then the notation $\operatorname{Hom}(S, X)$ denotes the semigroup of homomorphisms of $S$ into $X$ under the usual operation.

At the end of $\S 1$ we show that if $S$ is a CCIF-semigroup, $\operatorname{Hom}(S, \boldsymbol{R}) \neq\{0\}$, and the homomorphism group is transitive in some sense. In Section 2 we shall try to generalize the representation of $\Re$-semigroups to CCIF-semigroups. It will be understood as the socalled Schreier's extension to build up complicated CCIF-semigroups from simpler CCIF-semigroups. Most of the results in [7] will be extended to CCIF-semigroups. In $\S 3$ we shall treat the important case, i.e., the case where the structure semigroup is a group. Such

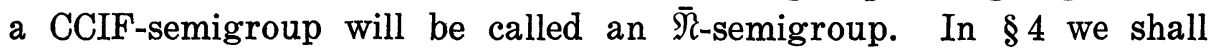
show that every CCIF-semigroup of finite rank has a nontrivial homomorphism into $\boldsymbol{R}_{+}^{0}$. In particular we will characterize CCIFsemigroups $S$ having the property $\operatorname{Hom}\left(S, \boldsymbol{R}_{+}\right) \neq \varnothing$.

(1.1) Let $S$ be a CCIF-semigroup. Then $x \neq x y$ for all $x, y \in S$. 
Proof. Suppose, for some $x, y \in S$, we have $x=x y$. Then $x y=$ $x y^{2}$ which implies $y=y^{2}$ by cancellation. This is a contradiction.

Proposition 1.2. Let $S$ be a CCIF-semigroup.

(1.2.1) Hom $(S, \boldsymbol{R})$ is a nontrivial vector space over the field $\boldsymbol{R}$.

(1.2.2) For each $a \in S$ and each $r \in \boldsymbol{R}, r \neq 0$, there is an $h \in \operatorname{Hom}(S, \boldsymbol{R})$ such that $h(a)=r$.

Proof of (1.2.1). Let $S$ be a CCIF-semigroup. Let $Q(S)$ be the quotient group of $S$ (i.e., the group of quotients of $S$ ), and $D(S)$ be the divisible hull of $Q(S)$

$$
D(S)=\bigoplus_{\alpha \in \Gamma} R_{\alpha} \oplus \bigoplus_{p \in \Delta} C\left(p^{\infty}\right) .
$$

$D(S)$ is a direct sum of copies $R_{\alpha}$ of the group of rational numbers under addition and quasi-cyclic groups $C\left(p^{\infty}\right)$ with respect to prime number $p$. We view $S$ as a subsemigroup of $D(S)$. Let $\pi_{\alpha}$ be the projection of $D(S)$ upon $R_{\alpha}$ for each $\alpha \in \Gamma$. Let $x$ be an element of S. Suppose $\pi_{\alpha}(x)=0$ for each $\alpha \in \Gamma$. It follows that $x \in \bigoplus_{p \in \triangle} C\left(p^{\infty}\right)$, a torsion group. This is a contradiction as $x$ has infinite order. Thus, for some $\alpha_{0} \in \Gamma, \pi_{\alpha_{0}}(x) \neq 0$. Note that $\pi_{\alpha_{0}} \in \operatorname{Hom}(S, R)$ and is not the trivial homomorphism. It is obvious that $\operatorname{Hom}(S, R)$ is a vector space over $\boldsymbol{R}$ in the usual way.

Proof of (1.2.2). Let $a \in S$ and $r \in R$ be given. In establishing (1.2.1), we have shown that there exists $h_{1} \in \operatorname{Hom}(S, R)$ with $h_{1}(a) \neq 0$. Let $s=h_{1}(a)$. Now define $h$ by $h=(r / s) h_{1}$. Then $h(a)=r$, and $h \in \operatorname{Hom}(S, R)$.

2. Schreier Extension. We consider the following problem. Let $C$ be a CCI-semigroup and $\varepsilon$ be its identity. Given $C$, find all CCIF-semigroups $S$ such that there is a homomorphism $\mathscr{P}$ of $S$ onto $C$ satisfying the condition.

$$
\{x \in S \mid \mathscr{P}(x)=\varepsilon\} \cong Z_{+} \cdot
$$

In this section we shall show that $S$ always exists for every $C$ and shall describe $S$ in terms of elements of $C$, integers and a certain function of $C \times C$ into the integers. The extension $S$ is called a Schreier extension (of $Z_{+}$) by $C$. (The terminology is due to [5].) Schreier extension by $C$ is significant because we shall see that every CCIF-semigroup is isomorphic to a Schreier extension by some CCIsemigroup $C$.

Theorem 2.1. Let $C$ be a CCI-semigroup and $C_{1}$ a proper ideal 
of $C . \quad\left(C_{1}\right.$ can be empty.) Let $I: C \times C \rightarrow Z$ be a function which satisfies

(2.1.1) $I(\alpha, \beta) \in Z_{+}^{0}$ if $\alpha \beta \notin C_{1}$

(2.1.2) $I(\alpha, \beta)=I(\beta, \alpha)$

for all $\alpha, \beta \in C$

(2.1.3) $I(\alpha, \beta)+I(\alpha \beta, \gamma)=I(\alpha, \beta \gamma)+I(\beta, \gamma)$ for all $\alpha, \beta, \gamma \in C$

(2.1.4) $I(\varepsilon, \alpha)=1$ ( $\varepsilon$ the identity element of $C$ ) for all $\alpha \in C$. Given $C, C_{1}, I$, the set $\left(C, C_{1} ; I\right)$ with its operation is defined by

$$
\left(C, C_{1} ; I\right)=\left\{(x, \alpha) \in Z \times C ; x \in Z_{+}^{0} \text { if } \alpha \notin C_{1}\right\}
$$

(2.1.5) $(x, \alpha)(y, \beta)=(x+y+I(\alpha, \beta), \alpha \beta)$.

Then $\left(C, C_{1} ; I\right)$ is a CCIF-semigroup.

Conversely if $S$ is a CCIF-semigroup, then $\left(S \cong C, C_{1} ; I\right)$ for some $C, C_{1}, I$.

Proof. It is routine to prove that $\left(C, C_{1} ; I\right)$ is a commutative cancellative simigroup. To show idempotent-freeness, assume $(x, \alpha)^{2}=$ $(x, \alpha)$, that is, $\alpha^{2}=\alpha$ and $2 x+I(\alpha, \alpha)=x$. It follows that $\alpha=\varepsilon$ and $x+1=0$. Since $C_{1}$ is a proper ideal of $C, \varepsilon \notin C_{1}$, hence $x \geqq 0$ and we arrive at a contradiction.

Conversely assume that $S$ is a CCIF-semigroup. Let $a \in S$, and define a relation $\rho_{a}$ on $S$ by

(2.1.6) $x \rho_{a} y$ iff $a^{m} x=a^{n} y$ for some $m, n \in Z_{+}$.

It is easy to see that $\rho_{a}$ is a congruence relation. To show that $S / \rho_{a}$ is cancellative, assume $x z \rho_{a} y z$. Then $a^{m} x z=a^{n} y z$ for some $m, n \in Z_{+}$. Since $S$ is cancellative, we get $a^{m} x=a^{n} y$, i.e., $x \rho_{a} y$. Obviously $a x \rho_{a} x$ for all $x \in S$, that is, the $\rho_{a}$-class containing $a$ is the identity of $S / \rho_{a}$. Let $C=S / \rho_{a} . \quad C$ is a CCI-semigroup. In each $\rho_{a}$-class define $x \leqq_{a} y$ by $x=a^{m} y$ for some $m \in Z_{+}^{0}$ where $a^{0} y=y$. Because of cancellation, each $\rho_{a}$-class forms a chain with respect to $\leqq_{a}$. Let $T=\bigcap_{n=1}^{\infty} a^{n} S$ and let $C_{1}$ be the image of $T$ under the natural homomorphism $S \rightarrow C$. If $T \neq \varnothing$, it is a proper ideal of $S$ (since $a \notin T$ ) and thus $C_{1}$ is a proper ideal of $C$. Under the homomorphism $S \rightarrow C$ we have a partition of $S: S=\bigcup_{\xi \in C} S_{\xi}$. If $\xi \in C \backslash C_{1}, S_{\xi}$ contains a maximal element with respect to $\leqq_{a}$; but if $\xi \in C_{1}, S_{\xi}$ contains no maximal element. For each $\xi \in C$, define $p_{\xi}$ to be $a \leqq_{a}$-maximal element in $S_{\xi}$ if $\xi \in C \backslash C_{1}$, and $p_{\xi}$ to be arbitrarily chosen from $S_{\xi}$ if $\xi \in C_{1}$. Since $C_{1}$ is a proper ideal, $\varepsilon \notin C_{1}$, hence $p_{\varepsilon}=a$ because of (1.1). Then every element of $S$ has a unique expression

$$
x=a^{m} p_{\xi} \text { where } m \in Z \text { if } \xi \in C_{1} ; m \in Z_{+}^{0} \text { if } \xi \in C \backslash C_{1} .
$$

Define $I: C \times C \rightarrow Z$ as follows:

$$
p_{\alpha} p_{\beta}=a^{I(\alpha, \beta)} p_{\alpha B} .
$$


It is easy to see that $I$ satisfies (2.1.1), (2.1.2), (2.1.3) and (2.1.4). $S$ is isomorphic to $\left(C, C_{1} ; I\right)$ under the map $a^{m} p_{\xi} \mapsto(m, \xi)$.

The representation $\left(C, C_{1} ; I\right)$ of $S$ depends on the choice of $a$. The element $a$ is called the standard element of the representation $\left(C, C_{1} ; I\right)$ of $S . \quad S / \rho_{a}$ is called the structure CCI-semigroup of $S$ with respect to $a ; C$ is the structure CCI-semigroup of $\left(C, C_{1} ; I\right)$, and $(0, \varepsilon)$ is the standard element. A function $I: C \times C \rightarrow Z$ satisfying (2.1.1), (2.1.2), (2.1.3), (2.1.4) is called an $\mathscr{F}$-function on $\left(C, C_{1}\right)$.

THEOREM 2.2. Let $C$ be a CCI-semigroup, and $C_{1}$ be a proper ideal of $C . \quad\left(C_{1}\right.$ can be empty.) Assume that $\varphi: C \rightarrow \boldsymbol{R}$ satisfies

(2.2.1) $\varphi(\alpha)+\varphi(\beta)-\varphi(\alpha \beta) \in \begin{cases}Z & \text { if } \alpha \beta \in C_{1} \\ Z_{+}^{0} & \text { if } \alpha \beta \notin C_{1}\end{cases}$

(2.2.2) $\varphi(\varepsilon)=1$.

Given $C, \phi$, and $C_{1}$, define $\left(\left(C, C_{1} ; \phi\right)\right)$ by and

(2.2.3) $\left(\left(C, C_{1} ; \varphi\right)\right)=\left\{((x+\varphi(\alpha), \alpha)): \alpha \in C, x \in Z, x \in Z_{+}^{0}\right.$ if $\left.\alpha \notin C_{1}\right\}$

(2.2.4) $((x+\varphi(\alpha), \alpha))((y+\varphi(\beta), \beta))=((x+y+\varphi(\alpha)+\varphi(\beta), \alpha \beta))$.

Then $\left(\left(C, C_{1} ; \varphi\right)\right)$ is a CCIF-semigroup.

Conversely every CCIF-semigroup is isomorphic to $\left(\left(C, C_{1} ; \varnothing\right)\right)$ for some $C$, $\varnothing$ and $C_{1}$, that is, $\left(C, C_{1} ; I\right) \cong\left(\left(C, C_{1} ; \varnothing\right)\right)$ under $(x, \alpha) \rightarrow$ $((x+\varphi(\alpha), \alpha)), I(\alpha, \beta)=\varphi(\alpha)+\varphi(\beta)-\varphi(\alpha \beta)$.

Proof. Assume $S$ is a CCIF-semigroup. By Theorem 2.1, we let $S=\left(C, C_{1} ; I\right)$ for some $C, I, C_{1}$. By (1.2.2), there is an $h \in \operatorname{Hom}(S, R)$ such $h(0, \varepsilon) \neq 0$. Define $\varphi: C \rightarrow \boldsymbol{R}$ by

(2.2.5) $\varphi(\alpha)=\frac{h(0, \alpha)}{h(0, \varepsilon)}$.

If $I(\alpha, \beta) \geqq 0$, then $(0, \alpha)(0, \beta)=(0, \varepsilon)^{I(\alpha, \beta)}(0, \alpha \beta)$ implies

$$
h(0, \alpha)+h(0, \beta)=I(\alpha, \beta) \cdot h(0, \varepsilon)+h(0, \alpha \beta) \text {. }
$$

If $I(\alpha, \beta)<0$, then $(0, \alpha)(0, \beta)(0, \varepsilon)^{-I(\alpha, \beta)}=(0, \alpha \beta)$ implies

$$
h(0, \alpha)+h(0, \beta)-I(\alpha, \beta) \cdot h(0, \varepsilon)=h(0, \alpha \beta) \text {. }
$$

In both cases, using (2.2.5), we have

(2.2.6) $I(\alpha, \beta)=\varphi(\alpha)+\varphi(\beta)-\varphi(\alpha \beta)$ for all $\alpha, \beta \in C$. It is easy to see that $\varphi$ satisfies (2.2.1) and (2.2.2); and $S=\left(C, C_{1} ; I\right) \cong\left(\left(C, C_{1} ; \varphi\right)\right)$ under $(x, \alpha) \mapsto((x+\varphi(\alpha), \alpha))$.

Conversely assume $\varphi$ satisfies (2.2.1) and (2.2.2), define $\left(\left(C, C_{1} ; \phi\right)\right)$ by (2.2.3) and (2.2.4), and define $I$ by (2.2.6). Then we can see that $I$ satisfies (2.1.1), (2.1.2), (2.1.3) and (2.1.4), and $((x, \alpha)) \mapsto(x-\varphi(\alpha), \alpha)$ gives an isomorphism of $\left(\left(C, C_{1} ; \varnothing\right)\right)$ to $\left(C, C_{1} ; I\right)$. 
A function $\varphi: C \rightarrow R$ is called a defining function on $\left(C, C_{1}\right)$ if it satisfies (2.2.1) and (2.2.2); let $\operatorname{Dfn}\left(C, C_{1}, \boldsymbol{R}\right)$ denote the set of all defining functions on $\left(C, C_{1}\right)$. If $\varphi$ satisfies (2.2.6) for a fixed $I, \varphi$ is called a defining function belonging to $I$, and the set of all $\phi$ belonging to $I$ is denoted by $\operatorname{Dfn}_{I}\left(C, C_{1}, \boldsymbol{R}\right)$.

Corollary 2.3. $S$ is a CCIF-semigroup if and only if $S$ is isomorphic to the subdirect product of a CCI-semigroup $C$ and $a$ subsemigroup of $\boldsymbol{R}$ by means of $\varphi$ on $C$ (i.e., by means of $\varphi$ with (2.2.1) and (2.2.2) in the sense of (2.2.4)).

CoROllaRY 2.4. Let $S$ be a CCIF-semigroup. $S$ is a subdirect product of a subsemigroup $P$ of $\boldsymbol{R}_{+}^{0}$ and a CCI-semigroup $C$ if and only if there exists $h \in \operatorname{Hom}\left(\left(S, \boldsymbol{R}_{+}^{j}\right)\right.$ with $h \neq 0$.

The problem posed at the beginning of the section is solved, that is,

$$
\mathscr{P}:((x+\varphi(\alpha), \alpha)) \longrightarrow \alpha
$$

has kernel $K=\left\{((x+1, \varepsilon)): x \in Z_{+}^{0}\right\}$ and $K \cong Z_{+}$under $((x+1, \varepsilon)) \rightarrow$ $x+1$.

Let $S=\left(C, C_{1} ; I\right)$.

Proposition 2.5. Let $\varphi_{0} \in \operatorname{Dfn}_{I}\left(C, C_{1}, R\right)$ be fixed. If $f \in \operatorname{Hom}(C, R)$ then $\varphi=\varphi_{0}+f \in \operatorname{Dfn}_{I}\left(C, C_{1}, \boldsymbol{R}\right)$. Every element $\varphi$ of $\operatorname{Dfn}_{I}\left(C, C_{1}, \boldsymbol{R}\right)$ can be obtained in this manner.

Proposition 2.6 (2.6.1). Let $\varphi_{0} \in \operatorname{Dfn}_{I}\left(C, C_{1}, R\right)$ be fixed and $f \in \operatorname{Hom}(C, \boldsymbol{R})$. Define $h: S \rightarrow \boldsymbol{R}$ by

$$
h(x, \alpha)=s\left(x+\varphi_{0}(\alpha)+f(\alpha)\right), \quad s \in \boldsymbol{R} .
$$

Then $h \in \operatorname{Hom}(S, \boldsymbol{R}) \quad$ Every element $h$ of $\operatorname{Hom}(S, \boldsymbol{R})$ satisfying $h(0, \varepsilon) \neq 0$ can be obtained in this manner.

(2.6.2) Let $p: S \rightarrow C$ be the natural homomorphism. Then every $h$ of $\operatorname{Hom}(S, \boldsymbol{R})$ satisfying $h(0, \varepsilon)=0$ is obtained by $h=f p$ where $f \in \operatorname{Hom}(C, \boldsymbol{R})$.

Proof (2.6.1). As the former half is easily proved, we prove the latter half. By (1.2.1) $\operatorname{Hom}(S, R) \neq\{0\}$, so there is $h$ such that $h(0, \varepsilon) \neq 0$. If $x \geqq 0$,

$$
\begin{aligned}
h(x, \alpha) & =h\left((0, \varepsilon)^{x}(0, \alpha)\right)=x \cdot h(0, \varepsilon)+h(0, \alpha) \\
& =h(0, \varepsilon)(x+\varphi(\alpha))=s(x+\varphi(\alpha))
\end{aligned}
$$


where $s=h(0, \varepsilon) ; \varphi(\alpha)=h(0, \alpha) / h(0, \varepsilon), \varphi \in \operatorname{Dfn}_{I}\left(C, C_{1}, R\right)$. If $x=0$, $(0, \varepsilon)^{x}$ is regarded as void. If $x<0,-x-1 \geqq 0$, then

$$
\begin{aligned}
h(0, \alpha) & =h((-x-1, \varepsilon)(x, \alpha))=h\left((0, \varepsilon)^{-x}(x, \alpha)\right) \\
& =(-x) \cdot h(0, \varepsilon)+h(x, \alpha)
\end{aligned}
$$

hence $h(x, \alpha)=h(0, \varepsilon)(x+\varphi(\alpha))$. By Proposition 2.5, $\varphi$ is expressed as $\varphi_{0}+f$. Thus we have the conclusion.

Proof. (2.6.2) Let $h \in \operatorname{Hom}(S, \boldsymbol{R})$ with $h(0, \varepsilon)=0$. If $x \geqq 0$, $h(x, \alpha)=x \cdot h(0, \varepsilon)+h(0, \alpha)=h(0, \alpha)$. If $x<0, h(0, \alpha)=(-x) \cdot h(0, \varepsilon)+$ $h(x, \alpha)=h(x, \alpha)$. Hence $h(x, \alpha)=h(0, \alpha)$ for all $(x, \alpha) \in S$. Define $f: C \rightarrow \boldsymbol{R}$ by $f(\alpha)=h(x, \alpha)$ where $(x, \alpha) \in S$. By the above result, $f$ is well defined. Now

$$
f p(x, \alpha)=f(\alpha)=h(x, \alpha), \text { hence } h=f p .
$$

It is easy to see that $f p \in \operatorname{Hom}(S, R)$ with $f p(0, \varepsilon)=0$.

By the notation $S=\left(C, C_{1} ; I\right)=\left(\left(C, C_{1} ; \varphi\right)\right)$ we mean that $S$ has representation $\left(C, C_{1} ; I\right)$ and $\left(\left(C, C_{1} ; \varphi\right)\right)$ identifying $(x, \alpha)$ of $\left(C, C_{1} ; I\right)$ with $((x+\varphi(\alpha), \alpha))$ of $\left(\left(C, C_{1} ; \varphi\right)\right)$.

Proposition 2.7. Let $S$ be a CCIF-semigroup. If $a \in S$ and if there is an $h \in \operatorname{Hom}\left(S, \boldsymbol{R}_{+}^{0}\right)$ such that $h(a) \neq 0$, then $C_{1}=\varnothing$ using $a$ as the standard element.

Proof. Let $S=\left(C, C_{1} ; I\right)=\left(\left(C, C_{1} ; \varphi\right)\right)$ and let $a$ denote $(0, \varepsilon)$ in $\left(C, C_{1} ; I\right)$ and at the same time $((1, \varepsilon))$ in $\left(\left(C, C_{1} ; \varphi\right)\right)$. Let $\alpha \in C_{1}$. Then $(x, \alpha) \in\left(C, C_{1} ; I\right)$ for all $x \in Z$. By Proposition 2.6

$$
h(x, \alpha)=h(0, \varepsilon)(x+\varphi(\alpha)) .
$$

Since $h(0, \varepsilon)>0$ and $x$ is arbitrary, $h(x, \alpha)<0$ if, $x<-\varphi(\alpha)$; a contradiction to the assumption. Hence $C_{1}=\varnothing$.

A subsemigroup $T$ of a commutative semigroup $S$ is called confinal if, for every $x \in S$, there is a $y \in S$ such that $x y \in T$. Let $S=\left(C_{1}, C ; I\right)$. The following are easily obtained.

LEMMA 2.8.

(2.8.1) If $C \backslash C_{1}$ contains a cofinal subsemigroup of $C$, then $C_{1}=\varnothing$.

(2.8.2) If $C$ is an abelian group, then $C_{1}=\varnothing$.

We will now make a further investigation into defining functions and $C_{1}$.

Let $U$ denote the group of units of $C$. Let $\varphi$ be a function 
$C \rightarrow \boldsymbol{R}$. Define a set $D_{C}(\varphi)$ by

$$
\begin{aligned}
D_{c}(\varphi)= & \{\alpha \in C: \varphi(\xi)+\varphi(\eta)-\varphi(\alpha)<0 \\
& \text { for some } \xi, \eta \in C \text { with } \alpha=\xi \eta\} .
\end{aligned}
$$

We define defining functions from the point of $C$.

\section{DEFINITION 2.9.}

(2.9.1) A function $\varphi: C \rightarrow \boldsymbol{R}$ is called a defining function on $C$ if it satisfies

$$
\left\{\begin{array}{l}
\varphi(\varepsilon)=1 . \\
\varphi(\alpha)+\varphi(\beta)-\varphi(\alpha \beta) \in Z \text { for all } \alpha, \beta \in C . \\
D_{C}(\varphi) \leqq C \backslash U .
\end{array}\right.
$$

The set of defining functions on $C$ is denoted by $\operatorname{Dfn}(C, R)$.

(2.9.2) A defining function on $C$ is called a normal defining function on $C$ if $D_{C}(\varphi)=\varnothing$, and a nonnormal defining function on $C$ if $D_{c}(\varphi) \neq \varnothing . \quad D_{C}(\varphi)$ is called the nonnormal domain of $\varphi$. The set of normal defining functions on $C$ is denoted by $\operatorname{NDfn}(C, R)$.

Proposition 2.10. Let $\varphi: C \rightarrow \boldsymbol{R}$ be a defining function on $C$. Let $C_{1}$ be a proper ideal of $C$ such that $D_{C}(\phi) \cong C_{1}$. Then $\varphi \in$ $\operatorname{Dfn}\left(C, C_{1}, \boldsymbol{R}\right)$. Conversely every defining function on $\left(C, C_{1}\right)$ is a defining function on $C$.

The following three cases are possible:

(i) $\varphi$ is normal and $C_{1}=\varnothing$

(ii) $\varphi$ is normal and $C_{1} \neq \varnothing$

(iii) $\varphi$ is not normal and $C_{1} \neq \varnothing$.

DEFINITION. In each case we consider the CCIF-semigroup $\left(\left(C, C_{1} ; \varphi\right)\right) . \quad\left(\left(C, C_{1} ; \varphi\right)\right)$ is called a normal representation in case (i); seminormal representation in case (ii); nonnormal representation in case (iii). In case (i), $\left(\left(C, C_{1} ; \varphi\right)\right)$ is denoted by $((C ; \varphi))$. When $\varphi$ is normal (nonnormal), the $\mathscr{F}$-function $I$ defined by $I(\alpha, \beta)=\varphi(\alpha)+$ $\varphi(\beta)-\varphi(\alpha \beta)$ is called normal (nonnormal); the corresponding semigroup is denoted by $\left(C, C_{1} ; I\right)$, in particular $(C ; I)$ in case (i).

Proposition 2.11. Let $S=\left(\left(C, C_{1} ; \varphi\right)\right)$ with standard element $a$. Then $\left(\left(C, C_{1} ; \varphi\right)\right)$ is a normal representation if and only if $\bigcap_{n=1}^{\infty} a^{n} S=\varnothing$.

Proposition 2.12. For every CCI-semigroup $C$ there exist normal defining functions on $C$. If $C$ is a CCI-semigroup and $C_{1}$ is a non- 
empty proper ideal of $C$, there exist nonnormal defining functions $\varphi$ such that the nonnormal domain of $\phi$ is contained in $C_{1}$.

Examples 2.13. Let $C$ be a CCI-semigroup.

(2.13.1) Define $\varphi$ by

$$
\varphi(\alpha)=1 \text { for all } \alpha \in C .
$$

Then $\varphi \in \operatorname{NDfn}(C, R)$, and $((C ; \varphi)) \cong Z_{+} \times C$.

(2.13.2) Let $U$ be the group of units of $C$. Let $\varphi_{0}$ be a nonnegative integer valued normal defining function on $U$. Define $\varnothing: C \rightarrow Z_{+}^{0}$ by

$$
\varphi(\alpha)= \begin{cases}\varphi_{0}(\alpha) & \text { if } \alpha \in U \\ c & \text { if } \alpha \notin U\end{cases}
$$

where $c$ is a constant nonnegative integer. Then $\varphi$ is a normal defining function on $C$.

(2.13.3) Let $C_{1}$ be a nonempty proper ideal of $C$. Define $\varphi$ by

$$
\varphi(\alpha)=\left\{\begin{aligned}
1 & \alpha \notin C_{1} \\
-1 & \alpha \in C_{1}
\end{aligned}\right.
$$

The $\varphi$ is a nonnormal defining function on $C$ such that $D_{C}(\varphi) \cong C_{1}$.

(2.13.4) Assume that $\varepsilon$ is the only unit of $C$. Suppose $\varphi_{0}: C \backslash\{\varepsilon\} \rightarrow R$ satisfies, for all $\alpha, \beta \in C \backslash\{\varepsilon\}$.

$$
\varphi_{0}(\alpha)+\varphi_{0}(\beta)-\varphi_{0}(\alpha \beta) \in Z \text {. }
$$

Define $\varphi: C \rightarrow \boldsymbol{R}$ by

$$
\varphi(\alpha)= \begin{cases}1 & \alpha=\varepsilon \\ \varphi_{0}(\alpha) & \alpha \neq \varepsilon\end{cases}
$$

Then $\phi$ is a defining function on $C$.

As another example, consider the case $C=Z_{+}^{0}$.

(2.14) Let $C=Z_{+}^{0}$. Let $\delta: Z_{+} \rightarrow Z$ be a function with $\delta(1)=0$ and let $r$ be a real number. Define $\varphi: Z_{+}^{0} \rightarrow \boldsymbol{R}$ by

$$
\varphi(m)=\left\{\begin{array}{ll}
1 & m=0 \\
m r-\delta(m) & m>0
\end{array} .\right.
$$

If $D_{z_{+}^{0}}(\varphi) \neq \varnothing$, take a proper ideal $C_{1}$ with $C_{1} \supseteqq D_{z_{+}^{0}}(\varphi)$. Then $\varphi \in$ $\operatorname{Dfn}\left(C, C_{1} ; R\right)$. Every defining function on $C$ is obtained in this manner. In particular if $\delta$ satisfies

$$
\delta(m)+\delta(n) \leqq \delta(m+n) \text { for all } m, n \in Z_{+},
$$


then $\varphi$ is a normal defining function on $C$.

We are interested in the important case, i.e., case where $C$ is a group. In the next section we discuss the structure of $((C, \varphi))$ where $C$ is a group. Then we will see that Example (2.14) is isomorphic to a Schreier extension by a group.

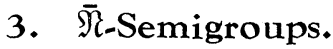

Definition 3.1. If $S$ is a commutative semigroup and $v \in S$ such that for all $x \in S$ there exist $m \in Z_{+}$and $y \in S$ with $v^{m}=x y$, then $S$ is called a subarchimedean semigroup and the element $v$ is called a pivot element of $S$.

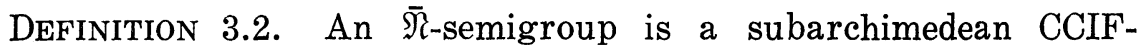
semigroup.

LEMMA 3.3. The pivot elements of a subarchimedean semigroup form an archimedean component and ideal of the semigroup.

Proof. Let $A$ be the set of pivot elements of a subarchimedean semigroup $S$. Let $v \in A$ and $x \in S$. There exist $m \in Z_{+}$and $y \in S$ such that $v^{m}=x y$. Then $(v z)^{m}=x\left(y z^{m}\right)$ for every $z \in S$; hence $v z \in A$. Thus $A$ is an ideal of $S$. To see that $A$ is archimedean, let $u, v \in A$. Then there exist $m \in Z_{+}$and $y \in S$ such that $v^{m}=u y$, therefore $v^{m+1}=u(y v)$ and $y v \in A$. Therefore $A$ is archimedean. Let $A_{0}$ be the archimedean component containing $v \in A$. Obviously $A \subseteq A_{0}$. Let $u \in A_{0}$, so $u^{n}=v y$ for some $n \in Z_{+}$, some $y \in S$. Let $z \in S$. As $v \in A, v^{k}=z t$ for some $k \in Z_{+}$, some $t \in S$. Then $u^{n k}=v^{k} y^{k}=z\left(t y^{k}\right)$, hence $u \in A, A_{0} \subseteq A$. Thus we have proved $A=A_{0}$.

LEMMA 3.4. A homomorphic image of a subarchimedean semigroup is a subarchimedean semigroup.

Proof. Let $S$ be a subarchimedean semigroup, and $f$ a surjective homomorphism of $S$ onto a semigroup $T$. Let $v$ be a privot element of $S$. Then for all $x \in S$ there exist $m \in Z_{+}$and $y \in S$ such that $v^{m}=x y$. Hence $(f(v))^{m}=f(x) f(y)$, and we see that $f(v)$ is a pivot element of $T$.

LEMMA 3.5. Let $S$ be a CCIF-semigroup. $S$ is subarchimedean if and only if $S / \rho_{a}$ is subarchimedean for (some) all $a \in S$.

Proof. If $S$ is subarchimedean then $S / \rho_{a}$ being a homomorphic image of $S$ is subarchimedean for all $a \in S$ by Lemma 3.4. Conversely, 
if $a \in S$ and $S / \rho_{a}$ is subarchimedean let $\bar{x}$ denote the $\rho_{a}$-class of $x \in S$. Let $\bar{v}$ be a pivot element of $S / \rho_{a}$. Then for all $\bar{x} \in S / \rho_{a}$ there exists $m \in Z_{+}$and $\bar{y} \in S / \rho_{a}$ such that $\bar{v}^{m}=\bar{x} \bar{y}$. Hence, by the definition of $\rho_{a}$ we have $v^{m} a^{k}=x y a^{l}$ for some $k, l \in Z_{+}$. Therefore, $(v a)^{m+k}=$ $x\left(y a^{l+m} v^{k}\right)$ and we see that $v a$ is a pivot element of $S$.

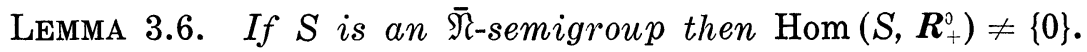

Proof. By Lemma 3.3, $S$ contains an $\mathfrak{R}$-semigroup $A$ which is an ideal of $S$. By $[2,7,8] \operatorname{Hom}\left(A, \boldsymbol{R}_{+}\right) \neq\{\varnothing\}$. Let $h \in \operatorname{Hom}\left(A, \boldsymbol{R}_{+}\right)$. Then $h \neq 0$. Define $\bar{h}: S \rightarrow \boldsymbol{R}$ by $\bar{h}(x)=h(a x)-h(a)$ for $a \in A$ and $x \in S$. Let $a, b \in A$, and $x \in S$. Then $h(a x)+h(b)=h((a x) b)=h((b x) a)=$ $h(b x)+h(a)$, so $h(a x)-h(a)=h(b x)-h(b)$. Thus $\bar{h}$ is well defined. Also, $\quad \bar{h}(x y)=h\left(a^{2} x y\right)-h\left(a^{2}\right)=h(a x)-h(a)+h(a y)-h(a)=\bar{h}(x)+$ $\bar{h}(y)$, hence $\bar{h}$ is a homomorphism. If $\bar{h}(x)<0$ for some $x \in S$, choose $n \in Z_{+}$such that $h(a)+n \bar{h}(x)<0$. Since $a x^{n} \in A, h\left(a x^{n}\right)>0$, but $h\left(a x^{n}\right)=h(a)+n \bar{h}(x)<0$, a contradiction. Hence $\bar{h} \in \operatorname{Hom}\left(S, \boldsymbol{R}_{+}^{0}\right)$. As $\bar{h} \mid A=h \neq 0$, $\operatorname{Hom}\left(S, \boldsymbol{R}_{+}^{0}\right) \neq\{0\}$.

Lemma 3.7. Let $S$ be an $\overline{\mathfrak{R}}$-semigroup. Then $a \in S$ is a pivot element if and only if $S / \rho_{a}$ is an abelian group.

Proof. Let $A$ be the archimedian ideal of pivot elements of $S$, and let $a \in A$. Then $A /\left(\rho_{a} \mid A\right)$ is an abelian group, and for all $x \in S$ we have $(x, x a) \in \rho_{a}$ where $x a \in A$. Hence $S / \rho_{a} \cong A /\left(\rho_{a} \mid A\right)$ and $S / \rho_{a}$ is an abelian group. Conversely if $S / \rho_{a}$ is an abelian group then for all $x \in S$ there exists $y \in S$ such that $\bar{a}=\bar{x} \bar{y}$ in $S / \rho_{a}$. (See the notation in the proof of Lemma 3.5.) Thus $a^{m}=x y a^{l}$ for some $m, l \in Z_{+}$. Hence $a \in A$.

THEOREM 3.8. Let $S$ be a CCIF-semigroup, and for $a \in S$ let $\rho_{a}$ be defind by (2.1.6). The following are equivalent:

(3.8.1) $S$ is an $\overline{\mathfrak{R}}$-semigroup.

(3.8.2) $S / \rho_{a}$ is subarchimedean for all $a \in S$.

(3.8.3) $S / \rho_{a}$ is subarchimedean for some $a \in S$.

(3.8.4) Some archimedean component of $S$ is an ideal of $S$.

(3.8.5) $S / \rho_{a}$ is an abelian group for some $a \in S$.

(3.8.6) $S \cong(G ; I)$ where $G$ is an abelian group and $I$ is an $\mathscr{F}$-function on $G$.

(3.8.7) $S$ is isomorphic to a subdirect product of an abelian group $G$ and a subsemigroup of $\boldsymbol{R}_{+}^{0}$ by means of a defining function $\varphi$ on $G$.

Proof. By Lemma 3.5, the first three conditions are equivalent. 
By Lemma 3.7, (3.8.1) implies (3.8.5); obviously (3.8.5) implies (3.8.3). By Lemma 3.3 and Lemma 3.7, (3.8.5) implies (3.8.4). Assume (3.8.4). Let $I$ be the ideal and archimedean component, and let $a \in I, x \in S$. Since $a x \in I, a^{m}=a x y$ for some $m \in Z_{+}$and some $y \in I$, hence $a^{m}=x(a y)$, that is, $a$ is a pivot element of $S$. By Lemma 3.7, (3.8.5) holds. By Theorem 2.1 and Lemma 2.8, (3.8.5) implies (3.8.6). Conversely if $S \cong(G ; I)$, then $G \cong S / \rho_{(0, \varepsilon)}$. Thus the first six conditions are equivalent. To see that (3.8.1) and (3.8.6) imply (3.8.7), let $S$ be an $\overline{\mathfrak{A}}_{\text {-semigroup. }}$ By Lemma 3.6 , there exists a nontrivial homomorphism $h$ of $S$ into $\boldsymbol{R}_{+}^{0}$, and by (3.8.6), $S \cong(G ; I)$ for some abelian group $G$ and an $\mathscr{F}$-function $I$. Let $\varphi(\alpha)=h(0, \alpha) / h(0, \varepsilon)$ for all $\alpha \in G$. (Clearly we can assume $h(0, \varepsilon) \neq 0$.) Then by the proof of Theorem 2.2 we have (3.8.7). Finally if we assume $(3.8 .7), S \cong((G ; \phi))$ for some $\varphi: G \rightarrow \boldsymbol{R}_{+}^{0}$, then when we define $I(\alpha, \beta)=\varphi(\alpha)+\varphi(\beta)-\varphi(\alpha, \beta)$, we have $S \cong(G ; I)$ as before. Hence (3.8.7) implies (3.8.6). The proof has been completed.

Corollary 3.9. Let $S$ be a CCIF-semigroup. $S$ is an $\Re$-semigroup if and only if $S / \rho_{a}$ is an abelian group for all $a \in S$.

Proof. Let $A$ be the set of pivot elements of $S$. If $S$ is an $\Re$-semigroup then $S=A$ and so $S / \rho_{a}$ is an abelian group for all $a \in S$. Conversely if $S / \rho_{a}$ is an abelian group for all $a \in S$ then $S=A$ by Lemma 3.7. Hence $S$ is archimedian, hence an $\mathfrak{N}$-semigroup.

4. Homomorphisms into $\boldsymbol{R}_{+\cdot}^{0}$. As seen in $\S 3$ every $\overline{\mathfrak{S}_{1}}$ semigroup has a nontrivial homomorphism into $\boldsymbol{R}_{+}^{\circ}$. The following question is raised.

Is a CCIF-semigroup nontrivially homomorphic into $\boldsymbol{R}_{+}^{\circ}$ ? We cannot answer this question in general, but in some special case it is affirmative.

Let $S$ be a CCIF-semigroup. As defined in $\S 1, Q(S)$ denotes the quotient group and $D(S)$ the divisible hull of $Q(S)$.

$$
D(S) \cong \bigoplus_{p \in \Delta} C\left(p^{\infty}\right) \bigoplus \bigoplus_{\alpha \in \Gamma} R_{\alpha}
$$

where $R_{\alpha}$ is a copy of the additive group of rationals and $C\left(p^{\infty}\right)$ is a quasicyclic group. The cardinality $|\Gamma|$ of $\Gamma$ is called the rank of $S$. In the present case the rank of $S$ is not zero since $\bigoplus_{p \in \Delta} C\left(p^{\infty}\right)$ is torsion while $S$ is torsion-free.

In particular, assume that $S$ is of finite rank. Let $T$ be the torsion subgroup of $D(S)$, then $D(S)=T \oplus R_{1} \oplus \cdots \oplus R_{n}$ where $n$ is 
the rank of $S$. We can assume $R_{i} \neq\{0\}$ for $i=1, \cdots, n$. Let $P_{i}=$ $R_{1} \oplus \cdots \oplus R_{i}$ for each $i=1,2, \cdots, n$. Then $P_{n}=P_{n-1} \oplus R_{n}$ if $n>1$; and $D(S)=T \oplus P_{n}$ if $n \geqq 1$. Let $\alpha, \bar{\sigma}, \sigma, \pi_{n}, \tau_{n}$ be the respective projection homomorphisms:

$$
\begin{gathered}
\alpha: D(S) \longrightarrow T, \quad \bar{\sigma}: D(S) \longrightarrow P_{n}, \quad \sigma=\bar{\sigma} \mid S, \\
\pi_{n}: P_{n} \longrightarrow P_{n-1}, \quad \tau: P_{n} \longrightarrow R_{n} \quad(n \geqq 1)
\end{gathered}
$$

THEOREM 4.1. If $S$ is a CCIF-semigroup of finite rank, then $\operatorname{Hom}\left(S, R_{+}^{0}\right) \neq\{0\} . \quad\left(R_{+}^{0}\right.$ is the additive semigroup of nonnegative rationals.)

Proof. $S$ is viewed as a subsemigroup of $D(S)$. We will prove the theorem by induction on $n$. Let $V_{n}=\pi_{n} \sigma(S), W_{n}=\tau_{n} \sigma(S), V=\sigma(S)$, $T^{\prime}=\alpha(S)$. As $D(S)=T \oplus P_{n}$, we have

$$
S=T^{\prime} \oplus_{s} V \text {, and if } n>1, V=V_{n} \oplus_{s} W_{n},
$$

where $\bigoplus_{s}$ denotes a subdirect sum, $V \subseteq P_{n}, V_{n} \subseteq P_{n-1}, W_{n} \subseteq R_{n}$, and $T^{\prime} \subseteq T$, hence $T^{\prime}$ is a torsion group. First we prove

\section{(4.1.1) $\quad V$ does not contain 0 .}

Suppose $V$ contains 0 . There is $x \in T^{\prime}$ such that $(x, 0) \in S$. Since $T^{\prime}$ is a torsion group, $m x=0$ for some $m \in Z_{+}$. Then $(0,0)=(x, 0)^{m} \in S$. This is a contradiction as $S$ has no idempotent.

In case $n=1, S=T^{\prime} \bigoplus_{s} W_{1}$ where $W_{1}=V \subset R_{1}$. By (4.1.1), $W_{1}$ must be isomorphic to a positive rational semigroup $R_{1}^{\prime}$, say, under $f$, i.e., $f\left(W_{1}\right)=R_{1}^{\prime}$, hence $f \tau_{1} \sigma \in \operatorname{Hom}\left(S, R_{+}^{0}\right) \backslash\{0\}$.

Assume $n>1$ and that the theorem holds for all semigroups of rank $i$ such that $i \leqq n-1$. As denoted above,

$$
S=T^{\prime} \bigoplus_{s} V, \quad V=V_{n} \bigoplus_{s} W_{n}
$$

where $V_{n} \subseteq P_{n-1}, W_{n} \subseteq R_{n}$. We can assume $V_{n} \neq\{0\}$, otherwise it is reduced to the case $n=1$.

If $V_{n}$ is a CCIF-semigroup, $V_{n}$ has a nontrivial homomorphism $f$ from $V_{n}$ into $R_{+}^{0}$ by the induction assumption, hence $f \pi_{n} \sigma \in$ $\operatorname{Hom}\left(S, R_{+}^{0}\right) \backslash\{0\}$.

If $V_{n}$ is a CCI-semigroup which is not a group, then $V_{n}=V_{n}^{\prime} \cup H$ where $V_{n}^{\prime} \neq \varnothing, H \neq \varnothing, V_{n}^{\prime}$ is an ideal of $V_{n}$ and it is a CCIF-semigroup, and $H$ is a group. Define $S^{\prime}$ by $S^{\prime}=\left(\left(\pi_{n} \sigma\right)^{-1}\left(V_{n}^{\prime}\right)\right) \cap S$ and $W_{n}^{\prime}=\tau_{n} \sigma\left(S^{\prime}\right)$. Then $S^{\prime}$ is an ideal of $S$ and

$$
S^{\prime}=V_{n}^{\prime} \oplus_{s} W_{n}^{\prime}
$$

By the preceding paragraph, Hom $\left(S^{\prime \prime}, R_{+}^{0}\right)$ contains a nontrivial 
element $f$. However, since $S^{\prime}$ is an ideal of $S, f$ can be extended to $\bar{f} \in \operatorname{Hom}\left(S, R_{+}^{0}\right)$. In fact $\bar{f}$ is obtained by defining $\bar{f}(x)=f(a x)-f(a)$ where $x \in S, a \in S^{\prime}$. It is easy to show that $\bar{f}$ is well defined and a homomorphism. Suppose $\bar{f}\left(x_{1}\right)<0$ for some $x_{1} \in S$. There exists $m \in Z_{+}$such that $m \bar{f}\left(x_{1}\right)+f(a)<0$. However

$$
m \bar{f}\left(x_{1}\right)+f(a)=f\left(a x_{1}^{m}\right) \geqq 0
$$

since $a x_{1}^{m} \in S^{\prime}$. This contradicts the assumption. Therefore $\bar{f}(x) \geqq 0$ for all $x \in S$. Hence $\operatorname{Hom}\left(S, R_{+}^{0}\right) \neq\{0\}$. Assume $V_{n}$ is a group. Let $\bar{W}_{n}=\left\{(0, z): z \in W_{n}\right\} \cap V$. It is obvious that $\bar{W}_{n}$ is a subsemigroup if $\bar{W}_{n} \neq \varnothing$. If $x \in V, x$ has the form $x=\left(x_{1}, x_{2}\right) \in V_{n} \oplus_{s} W_{n}, x_{1} \in V_{n}$, $x_{2} \in W_{n}$. Since $V_{n}$ is a group, there exists $y_{2} \in W_{n}$ such that $y=$ $\left(-x_{1}, y_{2}\right) \in V$. Then $x y=\left(0, x_{2}+y_{2}\right) \in \bar{W}_{n}$. This proves that $\bar{W}_{n} \neq \varnothing$ and it is cofinal in $V$. Suppose $x \in V$ and $a, x a \in \bar{W}_{n}$. We write $x=\left(x_{1}, x_{2}\right), a=\left(0, a_{2}\right)$ viewing them as in $V_{n} \bigoplus_{s} W_{n}$. Then $x a=$ $\left(x_{1}, x_{2}+a_{2}\right) \in \bar{W}_{n}$ implies $x_{1}=0$, hence $x \in \bar{W}_{n}$. Thus $\bar{W}_{n}$ is unitary in $V$. Since $\bar{W}_{n}$ does not contain $(0,0)$ by $(4.1 .1), \bar{W}_{n}$ is isomorphic to a positive rational semigroup $R_{n}^{\prime}$ under $f: \bar{W}_{n} \rightarrow R_{n}^{\prime}$. By (4.1.2) below, $f$ extends to $\bar{f} \in \operatorname{Hom}\left(V, R_{+}^{0}\right)$. Therefore $\bar{f} \sigma \in \operatorname{Hom}\left(S, R_{+}^{0}\right) \backslash\{0\}$.

(4.1.2) Let $S$ be a CCIF-semigroup and let $U$ be a unitary cofinal subsemigroup of $S$. Then every homomorphism of $U$ into $R_{+}^{\circ}$ extends to a homomorphism of $S$ into $R_{+}^{0}$.

This is immediately obtained from [4].

The proof of Theorem 4.1 has been completed.

REMARK 4.2. Let $S=R_{+} \oplus\left(\bigoplus_{\alpha \in \Gamma} R_{\alpha}\right)$ where $|\Gamma|=\infty, R_{\alpha}$ is the group of rationals. We note that $\operatorname{Hom}\left(S, R_{+}^{0}\right) \neq\{0\}$, yet $S$ is not of finite rank. Thus the converse of Theorem 4.1 does not hold.

Next we consider the relation between nontriviality of $\operatorname{Hom}\left(S, R_{+}^{0}\right)$ and the property

$$
\bigcap_{n=1}^{\infty} a^{n} S=\varnothing \quad \text { for some } a \in S \text {. }
$$

Proposition 4.4. If $\operatorname{Hom}\left(S, \boldsymbol{R}_{+}^{0}\right) \neq\{0\}$, then there is an element $a \in S$ satisfying (4.3).

Proof. Let $h \in \operatorname{Hom}\left(S, \boldsymbol{R}_{+}^{0}\right), h \neq 0$. There is $a \in S$ such that $h(a) \neq 0$. Choose $a$ as a standerd element. We have $C_{1}=\varnothing$ by Proposition 2.7 and then have (4.3) by Proposition 2.11 .

The converse of Proposition 4.4 is still open. 
Problem 4.5. Let $S$ be a CCIF-semigroup. If $\bigcap_{n=1}^{\infty} a^{n} S=\varnothing$ for some $a \in S$, then is the following true

$$
\operatorname{Hom}\left(S, \boldsymbol{R}_{\vdash}^{0}\right) \neq\{0\} ?
$$

However, we give a few examples with respect to the related problems.

EXAmpLE 4.6. Let $\bigcap_{n=1}^{\infty} a^{n} S=\varnothing$. There does not necessarily exist $h \in \operatorname{Hom}\left(S, \boldsymbol{R}_{+}^{0}\right)$ such that $h(a) \neq 0$.

Let $S=\left(\left(Z_{+}^{0} ; \varphi\right)\right)$ where $\varphi: Z_{+}^{0} \rightarrow Z$ is defined by

$$
\varphi(m)=1-m^{2} \text {. }
$$

It can be easily shown that $\varphi$ is a normal defining function on $Z_{+}^{0}$, and that if $a=((1,0)), \bigcap_{n=1}^{\infty} a^{n} S=\varnothing$. Every element $f_{t}$ of $\operatorname{Hom}\left(Z_{+}^{0}, \boldsymbol{R}\right)$ has the form

$$
f_{t}(m)=t m
$$

but there is no $t$ satisfying

$$
\varphi(m)+f_{t}(m)=1-m^{2}+t m \geqq 0 \text { for all } m \in Z_{+}^{0} .
$$

By Proposition 2.6, (2.6.1), there is no $h \in \operatorname{Hom}\left(S, \boldsymbol{R}_{+}^{0}\right)$ with $h(a) \neq 0$. However the projection $h_{0}: S \rightarrow Z_{+}^{0}$ is a nontrivial element of $\operatorname{Hom}\left(S, \boldsymbol{R}_{+}^{0}\right)$ such that $h_{0}(a)=0$. Thus $\operatorname{Hom}\left(S, \boldsymbol{R}_{+}^{0}\right) \neq\{0\}$ and so Example 4.6 is not a counterexample to the converse of Proposition 4.4. In fact the semigroup $S$ is an $\overline{\mathfrak{A}}$-semigroup.

ExAmPLE 4.7. We exhibit an example of a CCIF-semigroup $S$ which satisfies

$$
\bigcap_{n=1}^{\infty} a^{n} S \neq \varnothing \text { for all } a \in S,
$$

and hence $\operatorname{Hom}\left(S, \boldsymbol{R}_{+}^{0}\right)=\{0\}$.

$$
\text { Let } S=\left\{\left(a_{1}, \cdots, a_{m}\right): m, a_{m} \in Z_{+}, a_{i} \in Z, 1 \leqq i<m\right\}
$$

and define a binary operation on $S$ as follows: if $m \leqq n$,

$$
\begin{gathered}
\left(a_{1}, \cdots, a_{m}\right)\left(b_{1}, \cdots, b_{n}\right)=\left(b_{1}, \cdots, b_{n}\right)\left(a_{1}, \cdots, a_{m}\right) \\
=\left(a_{1}+b_{1}, \cdots, a_{m}+b_{m}, b_{m+1}, \cdots, b_{n}\right) .
\end{gathered}
$$

Then, with this product, $S$ is a CCIF-semigroup. Let $S_{1}=Z_{+}$and $S_{i}=Z^{i-1} \times Z_{+}$for $i>1$. Then $S$ is the union of the infinite chain of $S_{i}^{\prime}$ 's, $S=\bigcup_{i=1}^{\infty} S_{i}$ and $S_{i} S_{j} \subseteq S_{j}$ if $i \leqq j$. If $a \in S_{m}$ then

$$
\bigcap_{n=1}^{\infty} a^{n} S=\bigcup_{i>m} S_{i} \text {. }
$$


DEFinition 4.8. A semigroup $S$ is called an $\mathfrak{R}^{\prime}$-semigroup if $S$ is isomorphic to a subsemigroup of an $\mathfrak{\Re}$-semigroup.

THEOREM 4.9. Let $S$ be a CCIF-semigroup. $S$ is an $\mathfrak{N}^{\prime}$-semigroup if and only if

$$
\operatorname{Hom}\left(S, \boldsymbol{R}_{+}\right) \neq \varnothing
$$

Proof. Assume that $S$ is a subsemigroup of an $\mathfrak{R}$-semigroup $T$. By $[6,7]$ there is an $h \in \operatorname{Hom}\left(T, \boldsymbol{R}_{+}\right)$. Let $h_{1}$ be the restriction of $h$ to $S$. Then $h_{1} \in \operatorname{Hom}\left(S, \boldsymbol{R}_{+}\right)$.

Conversely let $\operatorname{Hom}\left(S, \boldsymbol{R}_{+}\right) \neq \varnothing$. By Proposition 2.7, $C_{1}=\varnothing$. By Theorem 2.2 and its Corollaries, $S \cong(C ; \varphi)$ where $C$ is a CCIsemigroup and $\varphi \in \operatorname{DNfn}(C, R)$; and $S$ is isomorphic to a subdirect product of a subsemigroup $P$ of $\boldsymbol{R}_{+}$and $C, S \cong P \times{ }_{S} C$. Let $Q$ be the group of quotients of $C$. Then $P \times{ }_{S} C$ is a subsemigroup of the direct product $\boldsymbol{R}_{+} \times Q$, but the last direct product is an $\mathfrak{R}$ semigroup. Consequently $S$ is an $\mathfrak{N}^{\prime}$-semigroup.

The two concepts, $\overline{\mathfrak{R}}$-semigroup and $\mathfrak{N}^{\prime}$-semigroup, are independent of each other.

EXAMPLE 4.10. Let $S=Z_{+} \cup\left(Z \times Z_{+}\right)$. A binary operation is defined to be the same as Example 4.7, that is, $S$ is a subsemigroup

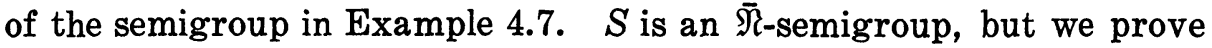
$\operatorname{Hom}\left(S, \boldsymbol{R}_{+}\right)=\varnothing$ as follows:

Let $x \in Z_{+}$and $\left(a_{1}, a_{2}\right) \in Z \times Z_{+}$. There exists $\left(b_{1}, b_{2}\right) \in Z \times Z_{+}$such that

$$
x \cdot\left(b_{1}, b_{2}\right)=\left(a_{1}, a_{2}\right) .
$$

Suppose $h \in \operatorname{Hom}\left(S, \boldsymbol{R}_{+}\right) \neq \varnothing$. Then

$$
h(x)<h\left(a_{1}, a_{2}\right) \text { for all } x \in Z_{+} \text {and all }\left(a_{1}, a_{2}\right) \in Z \times Z_{+} \text {. }
$$

In particular $h(1)<h\left(a_{1}, a_{2}\right)$, but there is $x \in Z_{+}$such that $x \cdot h(1)>$ $h\left(a_{1}, a_{2}\right)$. Accordingly $h(x)=x \cdot h(1)>h\left(a_{1}, a_{2}\right)$. This contradiction proves $\operatorname{Hom}\left(S, \boldsymbol{R}_{+}\right)=\varnothing$, hence $S$ is not an $\mathfrak{N}^{\prime}$-semigroup.

EXAMPLE 4.11. Let $S$ be the free commutative semigroup generated by infinitely countable letters $a_{1}, a_{2}, \cdots, a_{n}, \ldots$. (The empty word is not considered.) $S$ is obviously a CCIF-semigroup and $\operatorname{Hom}\left(S, R_{+}\right) \neq \varnothing$ since

$$
a_{i_{1}}^{m_{1}} \cdots a_{i_{k}}^{m_{k}} \longmapsto m_{1}+\cdots+m_{k}
$$

gives a homomorphism of $S$ into $Z_{+}$. However $S$ is not an $\overline{\mathfrak{R}}$-semi- 
group, as the greatest semilattice homomorphic image of $S$ does not have a zero.

REMARK. According to his recent personal letter to one of the authors, Professor Yuji Kobayashi, Tokushima University, has negatively answered Problem 4.5 by showing a counter example.

ACKNowledgment. The authors express their heart felt thanks to the referee of his kind advice to this paper.

\section{REFERENCES}

1. A. H. Clifford and G. B. Preston, Algebraic theory of semigroups, vol. 1, Amer. Math. Soc., Providence, Rhode Island, 1961.

2. Y. Kobayashi, Homomorphisms on $N$-semigroups into $R_{+}$and the structure of $N$-semigroups, J. Math. Tokushima University, 7 (1973), 1-20.

3. M. Petrich, Introduction to Semigroups, C. E. Merril Publ. Co., 1973.

4. M. S. Putcha and T. Tamura, Homomorphisms of commutative cancellative semigroups into nonnegative real numbers, to appear in Trans. Amer. Math. Soc.

5. L. Rédei, Die Verallgemeinerung der Schreierscher Erweiterungstheorie, Acta Sci. Math., Szeged., 14 (1952), 252-273.

6. T. Tamura, Commutative nonpotent archimedean semigroup with cancellation law. I., J. Gakugei Tokushima Univ., 8 (1957), 5-11.

7. - Basic study of $\mathfrak{R}$-semigroups and their homomorphisms, Semigroup Forum, 8 (1974), 21-50.

8. ——, Irreducible $\mathfrak{R}$ semigroups, Math. Nachrt., 63 (1974), 71-88.

Received March 19, 1975.

California State University, SaCramento

California State College, Stanislaus

AND

University of California, Davis, California 


\section{PACIFIC JOURNAL OF MATHEMATICS}

\section{EDITORS}

RICHARD ARENS (Managing Editor)

University of California

Los Angeles, California 90024

\author{
R. A. Beaumont \\ University of Washington \\ Seattle, Washington 98105
}

\section{J. DugundjI}

Department of Mathematics University of Southern California Los Angeles, California 90007

D. Gilbarg and J. Milgram

Stanford University

Stanford, California 94305

\section{ASSOCIATE EDITORS}
E. F. BECKENBACH
B. H. NeumanN
F. WOLF
K. YoshidA

\section{SUPPORTING INSTITUTIONS}

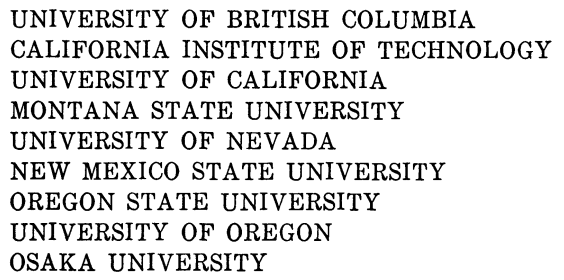

UNIVERSITY OF BRITISH COLUMBIA CALIFORNIA INSTITUTE OF TECHNOLOGY UNIVERSITY OF CALIFORNIA MONTANA STATE UNIVERSITY UNIVERSITY OF NEVADA NEW MEXICO STATE UNIVERSITY OSAKA UNIVERSITY OREGON STATE UNIVERSITY UNIVERSITY OF OREGON

\author{
UNIVERSITY OF SOUTHERN CALIFORNIA \\ STANFORD UNIVERSITY \\ UNIVERSITY OF TOKYO \\ UNIVERSITY OF UTAH \\ WASHINGTON STATE UNIVERSITY \\ UNIVERSITY OF WASHINGTON \\ AMERICAN MATHEMATICAL SOCIETY
}

The Supporting Institutions listed above contribute to the cost of publication of this Journal, but they are not owners or publishers and have no responsibility for its content or policies.

Mathematical papers intended for publication in the Pacific Journal of Mathematics should be in typed form or offset-reproduced, (not dittoed), double spaced with large margins. Please do not use built up fractions in the text of your manuscript. You may however, use them in the displayed equations. Underline Greek letters in red, German in green, and script in blue. The first paragraph or two must be capable of being used separately as a synopsis of the entire paper. Items of the bibliography should not be cited there unless absolutely necessary, in which case they must be identified by author and Journal, rather than by item number. Manuscripts, in triplicate, may be sent to any one of the editors. Please classify according to the scheme of Math. Reviews, Index to Vol. 39. All other communications should be addressed to the managing editor, or Elaine Barth, University of California, Los Angeles, California, 90024.

The Pacific Journal of Mathematics expects the author's institution to pay page charges, and reserves the right to delay publication for nonpayment of charges in case of financial emergency.

100 reprints are provided free for each article, only if page charges have been substantially paid. Additional copies may be obtained at cost in multiples of 50 .

The Pacific Journal of Mathematics is issued monthly as of January 1966. Regular subscription rate: $\$ 72.00$ a year $(6$ Vols., 12 issues). Special rate: $\$ 36.00$ a year to individual members of supporting institutions.

Subscriptions, orders for back numbers, and changes of address should be sent to Pacific Journal of Mathematics, 103 Highland Boulevard, Berkeley, California, 94708.

PUBLISHED BY PACIFIC JOURNAL OF MATHEMATICS, A NON-PROFIT CORPORATION

Printed at Kokusai Bunken Insatsusha (International Academic Printing Co., Ltd.), 8-8, 3-chome, Takadanobaba, Shinjuku-ku, Tokyo 160, Japan. 


\section{Pacific Journal of Mathematics}

\section{Vol. 61, No. 2 December, 1975}

Graham Donald Allen, Francis Joseph Narcowich and James Patrick Williams, An operator version of a theorem of Kolmogorov .......................

Joel Hilary Anderson and Ciprian Foias, Properties which normal operators share with normal derivations and related operators . . . . . . . . . . . . . . . . . . . . .

Constantin Gelu Apostol and Norberto Salinas, Nilpotent approximations and

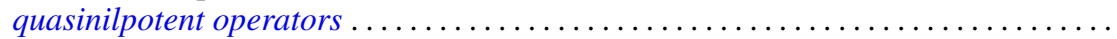

James M. Briggs, Jr., Finitely generated ideals in regular $F$-algebras . . . . . . . . . . .

Frank Benjamin Cannonito and Ronald Wallace Gatterdam, The word problem and power problem in 1-relator groups are primitive recursive ..................

Clifton Earle Corzatt, Permutation polynomials over the rational numbers ...........

L. S. Dube, An inversion of the $S_{2}$ transform for generalized functions . . . . . . . . . . William Richard Emerson, Averaging strongly subadditive set functions in unimodular

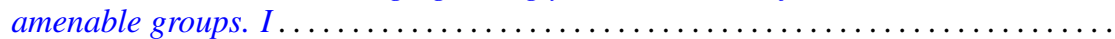

Barry J. Gardner, Semi-simple radical classes of algebras and attainability of

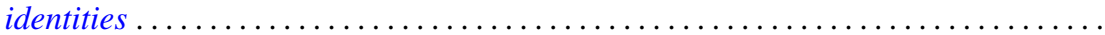

Irving Leonard Glicksberg, Removable discontinuities of A-holomorphic functions ....

Fred Halpern, Transfer theorems for topological structures . . . . . . . . . . . . . . .

H. B. Hamilton, T. E. Nordahl and Takayuki Tamura, Commutative cancellative

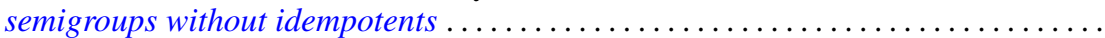

Melvin Hochster, An obstruction to lifting cyclic modules .....................

Alistair H. Lachlan, Theories with a finite number of models in an uncountable power

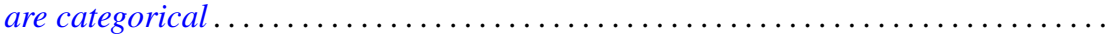

Kjeld Laursen, Continuity of linear maps from $C^{*}$-algebras . . . . . . . . . . . . .

Tsai Sheng Liu, Oscillation of even order differential equations with deviating arguments ....

Jorge Martinez, Doubling chains, singular elements and hyper- $Z$

Mehdi Radjabalipour and Heydar Radjavi, On the geometry of num Thomas I. Seidman, The solution of singular equations, I. Linear equations in Hilbert

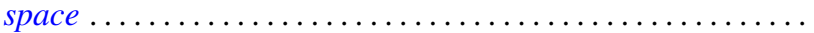

R. James Tomkins, Properties of martingale-like sequences ......

Alfons Van Daele, A Radon Nikodým theorem for weights on von Neumann

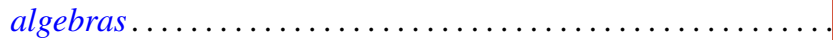

Kenneth S. Williams, On Euler's criterion for quintic nonresidues

Scott Andrew Wolpert, Noncompleteness of the Weil-Petersson metric for Teichmüller

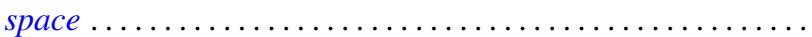

Volker Wrobel, Some generalizations of Schauder's theorem in locally convex

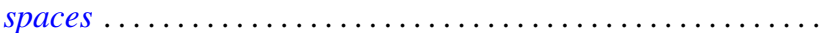

Kelly Denis McKennon, Corrections to: "Multipliers of type $(p, p)$ "; "Multipliers of type $(p, p)$ and multipliers of the group $L_{p}$-algebras"; "Multipliers and the

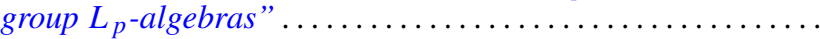

Andrew M. W. Glass, W. Charles (Wilbur) Holland Jr. and Stephen H. McCleary, Correction to: " $a *$-closures to completely distributive lattice-ordered

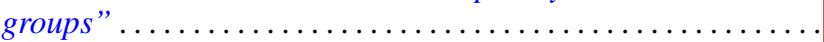

Zvi Arad and George Isaac Glauberman, Correction to: "A characteristic subgroup of

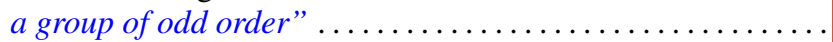

Roger W. Barnard and John Lawson Lewis, Correction to: "Subordination theorems

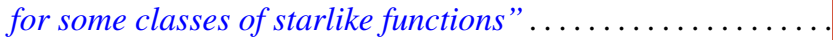

